\title{
Validation and comparative evaluation of four osteoporosis risk indexes in Moroccan menopausal women
}

\author{
Abdellah El Maghraoui • Amine Habbassi • \\ Mirieme Ghazi - Lahsen Achemlal • Aziza Mounach • \\ Abderrazak Nouijai • Ahmed Bezza
}

Received: 19 December 2005 / Accepted: 13 March 2006/ Published online: 22 August 2006

(C) International Osteoporosis Foundation and National Osteoporosis Foundation 2006

\begin{abstract}
Introduction Measuring bone mineral density (BMD) is a widely accepted strategy for identifying subjects with an increased risk of fracture. However, because of limited availability of BMD technology in some communities and cost considerations, it has been proposed that BMD measurements be targeted to subjects with risk factors for osteoporosis. Recently, many risk assessment indices have been developed to identify women who are more likely to have low BMD and thus undergo BMD testing. The objective of this study was to compare the performance of four risk indices for osteoporosis in white women in Morocco.

Methods We analysed in an epidemiological cross-sectional study the records for 986 postmenopausal white Moroccan women seen at an out-patient rheumatology centre. Four osteoporosis risk index scores were compared to bone density T-scores. The ability of each risk index to identify women with low BMD (T-score $<-2.0)$ or osteoporosis $(\mathrm{T}<-2.5)$ was evaluated.

Results Using an Osteoporosis Self-Assessment Tool (OST) score $<2$ to recommend DXA referral, we found that sensitivity ranged from $61 \%$ at the lumbar spine to $85 \%$ at the total hip to detect BMD T-scores of -2.5 , and specificity ranged from $62 \%$ at the lumbar spine to $67 \%$ at the total hip. The negative predictive value was high at all skeletal sites (79-98\%), demonstrating the usefulness of the OST to identify patients who have normal BMD and should not receive DXA testing. All risk indices performed
\end{abstract}

A. El Maghraoui $(\triangle) \cdot$ A. Habbassi $\cdot$ M. Ghazi $\cdot$ L. Achemlal

A. Mounach $\cdot$ A. Nouijai $\cdot$ A. Bezza

Rheumatology and Physical Rehabilitation Centre,

Military Hospital Mohammed V,

P.O. Box 1018, Rabat, Morocco

e-mail: abdellahe@menara.ma similarly and showed better results in identifying women with osteoporosis or low BMD based on hip measurement. Conclusions This is the first study that validated several risk osteoporosis indexes in Moroccan women. The performance of these risk indices among women in Morocco was similar to that reported earlier for other samples in Asian countries, the US, and Belgium. The OST and other risk indices are effective and efficient tools to help target high-risk women for DXA measurement.

Keywords Osteoporosis $\cdot$ Risk indices $\cdot$ Menopause · Morocco

\section{Introduction}

Osteoporosis is a systemic skeletal disorder characterized by low bone mass and micro-architectural deterioration of bone tissue, and as a consequence an increase in bone fragility and susceptibility to fracture [1]. It has enormous health and socioeconomic implications in terms of morbidity, mortality and disability worldwide [2]. In Morocco (30 million inhabitants), the age-adjusted 1-year cumulative incidence of hip fracture has been estimated to 52.1/100,000 [95\% confidence interval (CI) 40.9-63.3] in women in 2002 [3].

Bone mineral density (BMD) is widely recognized as the strongest predictor for future fracture occurrence, similar to an increased blood pressure level for the risk of stroke. Screening for low BMD by using dual energy $\mathrm{x}$-ray absorptiometry (DXA), the gold standard diagnostic tool for osteoporosis, is a widely accepted strategy for identifying subjects with an increased risk of fracture. However, mass screening with use of DXA scanning is not recommended without some selection of the target population. 
Furthermore, because of limited availability of BMD technology in some communities and cost considerations, it has been proposed that BMD measurements be targeted to subjects with risk factors for osteoporosis. Recently, many epidemiological studies have identified clinical risk factors for osteoporosis to develop risk assessment indices. The purpose of the risk assessment indices is not to diagnose osteoporosis or low BMD, but to identify women who are more likely to have low BMD. These patients can then be referred for BMD measurements. Such indices, while not identifying all cases of osteoporosis, increase the efficiency of BMD measurement by focusing on subjects who are at increased risk [4-7].

The easiest to use in clinical practice is certainly the Osteoporosis Self-assessment Tool (OST), which is based simply on age and weight [8]. It was developed and validated in several studies in Asian and white populations [9-13]. Other risk tools are also based on age and weight, in combination with up to four additional risk factors; these include the Osteoporosis Risk Assessment Instrument (ORAI) [14], the Simple Calculated Osteoporosis Risk Estimation (SCORE) [15], and the Osteoporosis Index of Risk (OSIRIS) [16]. These four risk assessment tools have been proposed for increasing awareness of osteoporosis and for encouraging more efficient use of BMD measurements in patients who are likely to have low bone mass, especially in asymptomatic postmenopausal women.

Our aims were to assess the validity of these risk indices in a population of 986 white women from Morocco, and to compare the performance of the four tools (OST, ORAI, SCORE and OSIRIS) in identifying women at risk of low BMD and who could benefit from definitive osteoporosis evaluation using DXA.

\section{Methods}

\section{Patients}

We analysed a database that included medical data on patients either consulted spontaneously or referred for a BMD measurement between April 2004 and July 2005 to our department located at the Rheumatology and Physical Rehabilitation Centre, Military Hospital Mohammed V (University of Rabat) in Morocco. Postmenopausal women $>45$ years at the date of consultation were included in our sample. Referral was based on diagnostic judgment of the referring physician. Informed consent was obtained from all eligible study participants. Patients with Paget's disease and advanced osteoarthritis were excluded. All participants underwent a structured questionnaire which concentrated on those variables for which published evidence suggested an association with osteoporosis or low BMD: current treatment for health complaints (including the presence of low back pain), fractures, presence of a disease, use of birth control pills, use of other hormones, ovariectomy, age of menopause, years between menarche and menopause (fertile years), number of pregnancies, family history of osteoporosis, use of toxic substances (alcohol and tobacco), calcium intake, and occupational exercise and sports in the present and past.

\section{Densitometry measurements}

All BMD measurements were performed on a Lunar Prodigy Vision machine (General Electric Inc). The DXA scans were obtained by standard procedures supplied by the manufacturer for scanning and analysis. The BMD measurements were carried out by two experienced technicians. Daily quality control was carried out by measurement of a Lunar phantom. At the time of the study, phantom measurements showed stable results. The phantom precision expressed as the CV (\%) was 0.08 . Moreover, reproducibility has been assessed recently in clinical practice and showed a smallest detectable difference of $0.04 \mathrm{~g} / \mathrm{cm}^{2}$ (spine) and 0.02 (hips) [17]. Patient BMD was measured at the lumbar spine (L1-4) (anteroposterior projection) and the femurs (dual femur). The mean result of the measure of the two femurs (total hip) was used. BMD values, expressed in $\mathrm{g} / \mathrm{cm}^{2}$, were converted into $\mathrm{T}$ scores, expressed in standard deviations (SDs), using French reference values. We used the WHO classification range to categorize subjects as normal $(\mathrm{T}>-1)$, osteopenic $(-2.5<$ $\mathrm{T} \leq-1)$, or osteoporotic $(\mathrm{T} \leq-2.5)$. A subcategory was defined as "low BMD", for all subjects with $\mathrm{T} \leq-2.0$, to allow comparison to published results for some risk indices that were based on this cut-off. Further, this cut-off is widely used in many communities to detect pre-osteoporotic patients.

Variables and risk scores

The OST, ORAI, SCORE, and OSIRIS indices were derived according to the algorithms suggested by their developers (Table 1). The following dichotomous cut-offs for DXA referral were used: $<2$ for OST, $>7$ for SCORE, $>8$ for ORAI and $<1$ for OSIRIS. Also, three risk categories were used for each index, according to their developer's recommendations and the validation of some indices in American and European populations.

Statistical analysis

Prevalence of osteoporosis in each of these three categories was determined using the WHO criteria. Receiver operating characteristic (ROC) analyses were performed to evaluate the discriminatory performances of OST, ORAI, SCORE, and OSIRIS, and the area under the curve (AUC) was 
Table 1 Method of calculation of the evaluated indices

\begin{tabular}{ll}
\hline Factor & Score \\
\hline OST (Osteoporosis Self-assessment Tool) & \\
Body weight $(\mathrm{kg})$ & $0.2 \times$ (body weight-age) \\
Age (years) & \\
SCORE (Simple Calculated Osteoporosis & Risk Estimation) \\
Race other than Black & +5 \\
Rheumatoid arthritis & +4 \\
Non-traumatic fracture after age & +4 per fracture, up to a \\
45 years & maximum of 12 \\
Age & +3 for each decade \\
Oestrogen therapy & +1 if never \\
Weight & -1 for each $10 \mathrm{lb}(4.5 \mathrm{~kg})$ \\
ORAI (Osteoporosis Risk Assessment Instrument) \\
Age $>75$ years & +15 \\
Age 65-74 years & +9 \\
Age 55-64 years & +5 \\
Body weight $<60 \mathrm{~kg}$ & +9 \\
Body weight $60-70 \mathrm{~kg}$ & +3 \\
Oestrogen therapy & +2 if not currently using \\
OSIRIS (Osteoporosis Index of Risk) & oestrogen \\
Body weight (kg) & $+0.2 \times$ body weight \\
Age (years) & $-0.2 \times$ age \\
History of low impact fracture(s) & -2 \\
Oestrogen therapy & +2 \\
\hline
\end{tabular}

computed for each. To assess the internal validity of the indices, sensitivity was defined as the proportion of the population with low BMD correctly classified by the risk index (true positive fraction) and specificity was defined as the proportion with normal BMD correctly identified by the risk index (true negative fraction). ROC curves provided a graphical representation of the overall accuracy of a test by plotting sensitivity against (1-specificity) for all thresholds, while the AUC quantified the accuracy of the test. We also calculated the positive predictive value (PPV) and negative predictive value (NPV) to evaluate the external validity of each tool. The PPV and NPV represent the proportion of women who tested positive or negative (as classified by the four tools) and who truly had, or did not have, BMD below the T-score threshold being tested, respectively.

We evaluated OST, ORAI, SCORE, and OSIRIS at the BMD T-score thresholds of -2.5 and -2.0 , to assess the performance of those indices in predicting osteoporosis and low bone mass, respectively. The ability of the tools to detect different thresholds of low BMD was also evaluated for various anatomical sites (total hip, femoral neck, L1-L4) of densitometric measurement. Statistical analysis used SPSS statistical software (SPSS Inc, Chicago, Ill).
Table 2 Characteristics of the participants $(n=986)$

\begin{tabular}{lllll}
\hline & Mean & SD & Minimum & Maximum \\
\hline Age (years) & 59.8 & 8.1 & 46 & 92 \\
Weight $(\mathrm{kg})$ & 71.8 & 12.9 & 35 & 118 \\
$\begin{array}{l}\text { Body mass index } \\
\left(\mathrm{kg} / \mathrm{m}^{2}\right)\end{array}$ & 35.6 & 6.3 & 17.7 & 56.6 \\
Spine BMD $\left(\mathrm{g} / \mathrm{cm}^{2}\right)$ & 0.962 & 0.168 & 0.526 & 1.617 \\
Spine T score & -1.67 & 1.4 & -5.30 & 3.80 \\
Femoral neck BMD & 0.887 & 0.14 & 0.360 & 1.145 \\
$\left(\mathrm{~g} / \mathrm{cm}^{2}\right)$ & & & & \\
Femoral neck T score & -1.16 & 1.08 & -4.30 & 3.20 \\
Total hip BMD $\left(\mathrm{g} / \mathrm{cm}^{2}\right)$ & 0.837 & 0.139 & 0.700 & 1.359 \\
Total hip T score & -0.92 & 1.160 & -4.30 & 3.80 \\
\hline
\end{tabular}

\section{Results}

The mean age of the women in our sample was $59.8( \pm 8.8)$ years, ranging from 46 to 92 years. Table 2 shows their basic demographic data. $15 \%$ reported a non-traumatic fracture after age 45 at the wrist, rib, or hip. The prevalence of osteoporosis at all sites increased progressively with age. Of the women in our study, 33\% were osteoporotic $(\mathrm{T}<-2.5)$ at one or more skeletal site, $45 \%$ had a low BMD ( $\mathrm{T}<-2.0)$, and $77 \%$ were classified as osteopenic according to the WHO operational definition. Table 3 shows the performance of the four risk indices in

Table 3 Prevalence of low BMD and osteoporosis by BMD measurement site and risk category

\begin{tabular}{|c|c|c|c|c|c|}
\hline \multirow[t]{2}{*}{ Risk category } & \multirow[t]{2}{*}{ Total } & \multicolumn{4}{|c|}{ T score $<2.5$} \\
\hline & & $\begin{array}{l}\text { Hip } \\
8.3 \%\end{array}$ & $\begin{array}{l}\mathrm{FN} \\
11.6 \%\end{array}$ & $\begin{array}{l}\text { L1-L4 } \\
31.1 \%\end{array}$ & $\begin{array}{l}\text { Any } \\
33.7 \%\end{array}$ \\
\hline \multicolumn{6}{|l|}{ OST } \\
\hline$>1$ (low risk) & $69.6 \%$ & $2.3 \%$ & $4.2 \%$ & $23.0 \%$ & $24.1 \%$ \\
\hline $\begin{array}{l}-3 \text { to } 1 \\
\text { (moderate risk) }\end{array}$ & $27.0 \%$ & $17.3 \%$ & $22.3 \%$ & $46.6 \%$ & $51.9 \%$ \\
\hline$<-3$ (high risk) & $3.4 \%$ & $58.3 \%$ & $73.5 \%$ & $73.5 \%$ & $85.3 \%$ \\
\hline \multicolumn{6}{|l|}{ SCORE } \\
\hline$<7$ (low risk) & $34.7 \%$ & $1.5 \%$ & $2.3 \%$ & $11.4 \%$ & $11.7 \%$ \\
\hline $\begin{array}{l}7-15 \\
\text { (moderate risk) }\end{array}$ & $59.4 \%$ & $8.4 \%$ & $11.9 \%$ & $39.2 \%$ & $42.2 \%$ \\
\hline$>15$ (high risk) & $5.9 \%$ & $48.3 \%$ & $62.1 \%$ & $65.5 \%$ & $77.6 \%$ \\
\hline \multicolumn{6}{|l|}{ ORAI } \\
\hline$<9$ (low risk) & $63.8 \%$ & $1.7 \%$ & $3.3 \%$ & $21.9 \%$ & $22.9 \%$ \\
\hline $\begin{array}{l}9-17 \\
\text { (moderate risk) }\end{array}$ & $29.5 \%$ & $13.4 \%$ & $19.2 \%$ & $44.7 \%$ & $47.4 \%$ \\
\hline$>17$ (high risk) & $6.7 \%$ & $48.5 \%$ & $56.1 \%$ & $59.1 \%$ & $75.8 \%$ \\
\hline \multicolumn{6}{|l|}{ OSIRIS } \\
\hline$>1$ (low risk) & $68.9 \%$ & $2.4 \%$ & $4.1 \%$ & $22.8 \%$ & $23.9 \%$ \\
\hline $\begin{array}{l}-3 \text { to } 1 \\
\text { (moderate risk) }\end{array}$ & $26.5 \%$ & $14.6 \%$ & $19.5 \%$ & $44.8 \%$ & $49.4 \%$ \\
\hline$<-3$ (high risk) & $4.7 \%$ & $60.9 \%$ & $76.1 \%$ & $76.1 \%$ & $89.1 \%$ \\
\hline
\end{tabular}


Table 4 Performance of the risk indices by BMD measurement site and T-score cut-off (\%)

\begin{tabular}{|c|c|c|c|c|c|c|c|c|c|c|c|c|c|c|c|c|}
\hline \multirow[t]{2}{*}{ Tool } & \multicolumn{4}{|c|}{ Total hip } & \multicolumn{4}{|c|}{ Femoral Neck } & \multicolumn{4}{|c|}{ L1-L4 spine } & \multicolumn{4}{|c|}{ Any site } \\
\hline & $\mathrm{Se}$ & $\mathrm{Sp}$ & PPV & NPV & $\mathrm{Se}$ & $\mathrm{Sp}$ & PPV & NPV & $\mathrm{Se}$ & $\mathrm{Sp}$ & PPV & NPV & $\mathrm{Se}$ & $\mathrm{Sp}$ & PPV & NPV \\
\hline \multicolumn{17}{|c|}{ T score $<2.5$} \\
\hline OST & 85 & 62 & 17 & 98 & 85 & 64 & 24 & 97 & 61 & 67 & 46 & 79 & 62 & 69 & 51 & 78 \\
\hline SCORE & 93 & 62 & 12 & 88 & 93 & 38 & 16 & 97 & 87 & 44 & 41 & 88 & 88 & 46 & 45 & 46 \\
\hline ORAI & 86 & 68 & 20 & 98 & 81 & 69 & 26 & 69 & 55 & 72 & 47 & 72 & 56 & 74 & 52 & 77 \\
\hline OSIRIS & 80 & 73 & 21 & 97 & 75 & 74 & 28 & 95 & 49 & 77 & 49 & 77 & 51 & 79 & 55 & 76 \\
\hline \multicolumn{17}{|l|}{ T score $<2$} \\
\hline OST & 76 & 67 & 35 & 92 & 70 & 67 & 38 & 88 & 55 & 65 & 61 & 70 & 57 & 66 & 63 & 71 \\
\hline SCORE & 94 & 40 & 12 & 94 & 90 & 42 & 31 & 93 & 82 & 49 & 57 & 77 & 83 & 77 & 58 & 50 \\
\hline ORAI & 70 & 72 & 37 & 91 & 67 & 72 & 41 & 88 & 50 & 75 & 63 & 64 & 52 & 65 & 66 & 77 \\
\hline OSIRIS & 66 & 77 & 41 & 90 & 61 & 77 & 44 & 87 & 43 & 79 & 63 & 62 & 45 & 63 & 66 & 80 \\
\hline
\end{tabular}

NPV: negative predictive value, PPV: positive predictive value, Se: sensitivity, Sp; specificity

identifying patients at various BMD measurement sites and thresholds ( $T$ score values of -2 and -2.5 ). Increasing prevalence of osteoporosis $(\mathrm{T}<-2.5)$ with ascending risk category (low, medium, high) was apparent for all four risk tools. For example, the prevalence of osteoporosis based on total hip BMD was approximately $2 \%, 17 \%$, and $58 \%$ at the low $(70 \%$ of women), medium ( $27 \%$ of women), and high ( $3 \%$ of women) OST risk levels. Of the women in our study classified as high risk, $91 \%$ had low bone mass and $85 \%$ had osteoporosis at any site. At the considered thresholds, OST, SCORE, ORAI, and OSIRIS identified respectively, $75 \%, 88 \%, 77 \%$, and $76 \%$ of the patients with normal BMD who subsequently should not have been recommended for densitometry, since according to their score they were 'low risk'.

Using the dichotomous cut-off value of $<2$, the sensitivity of OST in identifying individuals at increased risk of osteoporosis ranged from $82 \%$ for lumbar spine to $91 \%$ at the total hip region. The corresponding specificity of OST ranged from $24 \%$ at the total hip to $34 \%$ at any given site (Table 4). SCORE had a higher sensitivity than OST but lower specificity and NPV. At the OST cut-off point of 2,

Table 5 Areas under the ROC curves for the four evaluated assessment tools by BMD measurement site and T-score cut-off

\begin{tabular}{lllll}
\hline Tool & Total hip & Femoral neck & L1-L4 spine & Any site \\
\hline T score $<2.5$ & & & & \\
OST & 0.853 & 0.823 & 0.720 & 0.742 \\
SCORE & 0.851 & 0.820 & 0.681 & 0.723 \\
ORAI & 0.829 & 0.810 & 0.680 & 0.699 \\
OSIRIS & 0.850 & 0.820 & 0.721 & 0.735 \\
T score $<2$ & & & & \\
OST & 0.775 & 0.768 & 0.723 & 0.730 \\
SCORE & 0.768 & 0.759 & 0.679 & 0.695 \\
ORAI & 0.759 & 0.790 & 0.690 & 0.703 \\
OSIRIS & 0.780 & 0.780 & 0.716 & 0.745 \\
\hline
\end{tabular}

and using a BMD T-score threshold of -2.5 for any site, $51 \%$ of the subjects were misclassified (most of these were false positives); the proportion of misclassified patients for single BMD sites was $45 \%$ at the total hip, $32 \%$ at the femoral neck, and $49 \%$ at the lumbar spine site. The AUC was consistently high (around 0.8 ) for the two hip sites, and somewhat lower for the spine (Table 5), indicating good test performance. For each combination of BMD measurement and T-score cut-off, the AUC results were similar for all four risk tools.

\section{Discussion}

The National Institutes of Health consensus conference defined osteoporosis as a disease of increased skeletal fragility accompanied by low BMD (T score below -2.5 ) and micro-architectural deterioration [18]. The preferred sites for diagnostic purposes are BMD measurements made at the hip, either at the total hip or the femoral neck [19]. The availability of new effective treatments for osteoporosis emphasised to recommend BMD measurements for patients considered at high risk. Several guidelines have been developed to select which patients should undergo DXA testing [20-23].

In our study involving white women aged 45 years and more, the OST successfully identified most women with hip osteoporosis and low BMD with a sensitivity of $85 \%$, specificity of $62 \%$ and NPV of $98 \%$ at the total hip site. The OST, based only on age and weight, performed as well as the more complex risk assessment indices (SCORE, ORAI, and OSIRIS) in identifying women at low risk of osteoporosis who would not need DXA testing (98\% of patients classified as low risk with OST do not have osteoporosis at the total hip). In a population-based sample of postmenopausal Japanese women [10], the OST had a sensitivity of $90 \%$ and 
Table 6 Prevalence of postmenopausal women with osteoporosis by OST risk level in the current study and other published studies

\begin{tabular}{llllll}
\hline $\begin{array}{l}\text { OST risk } \\
\text { level }\end{array}$ & \multicolumn{5}{l}{ Prevalence of osteoporosis $(\mathrm{T}<-2.5)$ at the total hip } \\
\cline { 2 - 6 } & $\begin{array}{l}\text { Original Asian } \\
\text { sample, Koh et al. [8] }\end{array}$ & $\begin{array}{l}\text { Korea, Park } \\
\text { et al. [9] }\end{array}$ & $\begin{array}{l}\text { Belgium, } \\
\text { Richy et al. [12] }\end{array}$ & $\begin{array}{l}\text { The Philippines, } \\
\text { Li-Yu et al. [13] }\end{array}$ & $\begin{array}{l}\text { Morocco, } \\
\text { our study }\end{array}$ \\
\hline Low & $3 \%$ & $2 \%$ & $1.4 \%$ & $0.5 \%$ & $2.3 \%$ \\
Medium & $15 \%$ & $18 \%$ & $10.6 \%$ & $12.3 \%$ & $17.3 \%$ \\
High & $61 \%$ & $64 \%$ & $43.7 \%$ & $51.3 \%$ & $58.3 \%$ \\
\hline
\end{tabular}

specificity of $45 \%$. This tool was similarly validated in Korean [9], Filipino [12] and Belgian women [11]. Table 6 shows the prevalence of postmenopausal women with hip osteoporosis by OST risk level in the current study and other published studies.

Avoiding unnecessary testing among low risk patients can substantially reduce cost for the community and the patient (social health insurance only covers 15\% of Moroccan population). For example, in this sample of Moroccan women, $69 \%$ of the women were classified as low risk using OST, and thus would not need to be referred to DXA testing. Of these, only $2.3 \%$ actually had osteoporosis based upon total hip BMD. At the same time, using OST to select the $31 \%$ of women at risk for BMD measurements, $80 \%$ of all women with osteoporosis at the hip level would be identified.

A risk assessment tool such as OST does not need to have both high sensitivity and high specificity. Indeed, there is no risk of harm to the patient from unnecessary treatment or invasive diagnostic testing in case of a falsepositive result from OST. Although some women who do not have low BMD were classified as increased risk (false positives) and would be referred for testing, some of these women would have undergone testing anyway if OST were not used. Furthermore, treatment for low BMD would only be initiated upon confirmation by DXA: a safe and noninvasive diagnostic procedure.

Despite differences in ethnicity of the studied populations and the reference databases used to calculate T scores, we found the performance of OST in this sample similar to that reported among Asian, American and European women especially at the hip level. As it was the case in Richy et al. study [11], the results were slightly less concordant at the lumbar spine. We used the same risk tool categories as Geusens et al. [13] to compare the performance of the various tools in a different population, and found very similar results for OST at the T-score $\leq-2.0$ and $\leq-2.5$ cutoffs. The SCORE, ORAI, OSIRIS, and OST are validated risk indices that can help physicians and public health authorities to focus DXA testing on individuals at increased risk of osteoporosis. All four risk tools performed similarly, and identified a significant proportion of all women at low risk who would not benefit from BMD measurements.
These indices have been studied and validated in several different large populations.

As with most studies, our study has limitations. For example, the subjects in our sample were either referred or came in spontaneously for osteoporosis evaluations, and may differ in some ways from the general population. Another limit is the use of French BMD reference range for calculating T-scores. However, it was felt that awaiting the Moroccan reference database, now under study in our centre [24], the French population has more similarities with the Moroccan population than the US population used in NHANES III. Moreover, hip incidence measured in Morocco showed similar results to the south of France [3]. Another limitation of this kind of studies is that it does not take into account the risk of fracture, which is the main purpose of treating osteoporosis. DXA itself has a low sensitivity and about half of patients who fracture do not have densitometric osteoporosis. However, the main objective of our study and similar studies is to identify patients with low BMD in order to avoid unnecessary exams, which is very important in developing countries, while developing a fracture risk assessment tool needs prospective longitudinal cohorts.

In summary, measuring BMD is the best method of identifying patients with osteoporosis to consider for treatment. However, measuring BMD in all postmenopausal women is not feasible especially in developing countries such as Morocco. The OST tool can help target BMD measurements to women at risk. Its high sensitivity, acceptable specificity and very high negative predictive value allows for the safe exclusion of healthy women, in order to reduce unnecessary bone density examinations in those who are at lowest risk. ORAI, SCORE, and OSIRIS provided similar performance in this setting. The main advantage in using the OST index is that it is the simplest and quickest to calculate, and thus to use in general practice as a first-line prescreening tool in post-menopausal women.

\section{References}

1. NIH Consensus Development Conference (1993) Diagnosis, prophylaxis and treatment of osteoporosis. Am J Med 94:646-650 
2. Randell AG, Nguyen TV, Bhalerao N, Silverman SL, Sambrook PN, Eisman JA (2000) Deterioration in quality of life following hip fracture: a prospective study. Osteoporos Int 11:460-466

3. El Maghraoui A, Koumba BA, Jroundi I, Achemlal L, Bezza A, Tazi MA (2005) Epidemiology of hip fractures in 2002 in Rabat, Morocco. Osteoporos Int 16(6):597-602

4. Ribot C, Pouilles JM, Bonneau M, Tremollieres F (1992) Assessment of the risk of post-menopausal osteoporosis using clinical factors. Clin Endocrinol 36:225-228

5. Lydick E, Cook K, Turpin J, Melton M, Stine R, Byrnes C (1998) Development and validation of a simple questionnaire to facilitate identification of women likely to have low bone density. Am J Manag Care 4:37-48

6. Cadarette S, Jaglal S, Murray T et al. (2001) Evaluation of decision rules for referring women for bone densitometry by dualenergy x-ray absorptiometry. JAMA 286:57-63

7. van der Voort DJ, Dinant GJ, Rinkens PE, van der Voort CJ, va Mersch JW, Geusens PP (2000) Construction of an algorithm for quick detection of patients with low bone mineral density and its applicability in daily general practise. J Clin Epidemiol 53: 10951103

8. Koh LK, Ben Sedrine W, Torralba TP et al. (2001) Osteoporosis Self-Assessment Tool for Asians (OSTA) Research Group. A simple tool to identify Asian women at increased risk of osteoporosis. Osteoporos Int 12:699-705

9. Park HM, Ben Sedrine W, Reginster JY, Ross PD (2003) Korean experience with the OSTA risk index for osteoporosis. J Clin Densitom 6:251-258

10. Fujiwara S, Masunari N, Suzuki G et al. (2001) Performance of osteoporosis risk indices in a Japanese population. Curr Ther Res 62:586-594

11. Richy F, Gourlay M, Ross PD, Sen SS, Radican L, De Ceulaer L, Ben Sedrine W, Ethgen O, Bruyere O, Reginster JY (2004) Validation and comparative evaluation of the osteoporosis selfassessment tool (OST) in a Caucasian population from Belgium. Q J Med 97:39-46

12. Li-Yu JT, Llamado LJ, Torralba TP (2005) Validation of OSTA among Filipinos. Osteoporos Int Jul 19 [Epub ahead of print]

13. Geusens P, Hochberg MC, van der Voort DJ, Pols H, van der Klift M, Siris E (2002) Performance of risk indices for identifying low bone density in postmenopausal women. Mayo Clin Proc 77: 629-637

14. Cadarette SM, Jaglal SB, Kreiger N, McIsaac WJ, Darlington GA, Tu JV (2000) Development and validation of the osteoporosis risk assessment instrument to facilitate selection of women for bone densitometry. CMAJ 162:1289-1294

15. Ben Sedrine W, Devogelaer JP, Kaufman JM et al. (2001) Evaluation of the simple calculated risk estimation (SCORE) in a sample of white women from Belgium. Bone 29:374-380

16. Ben Sedrine W, Chevallier T, Zegels B et al. (2002) Development and assessment of the Osteoporosis Index of Risk (OSIRIS) to facilitate selection of women for bone densitometry. Gynecol Endocr 16:245-250

17. El Maghraoui A, Do Santos Zounon AA, Jroundi I et al. (2005) Reproducibility of bone mineral density measurements using dual $\mathrm{X}$-ray absorptiometry in daily clinical practice. Osteop Int 16 (12): $1742-1748$

18. (2001) NIH consensus development panel on osteoporosis prevention, diagnosis, and therapy. Osteoporosis prevention, diagnosis, and therapy. JAMA 285:785-795

19. Kanis JA, Glüer CC; for the Committee of Scientific Advisors, International Osteoporosis Foundation (2000) An update on the diagnosis and assessment of osteoporosis with densitometry. Osteoporos Int 11:192-202

20. (1999) Physician's guide to prevention and treatment of osteoporosis. National Osteoporosis Foundation, Washington DC

21. US Preventive Task Force (2002) Screening for osteoporosis in postmenopausal women: recommendations and rationale. Ann Int Med 137:526-528

22. National Osteoporosis Foundation (2003) Physician's guide to prevention and treatment of osteoporosis 2003. [http://www.nof.org/] Accessed 22 June 2003

23. (1999) Osteoporosis prevention, diagnosis and therapy consensus development conference. National Institutes of Health, 2000. 27. In: Genant HK, Cooper C, Poor G et al. (eds) Interim report and recommendations of the World Health Organization Task-Force for Osteoporosis. Osteoporos Int 102:343-350

24. El Maghraoui A, Guerboub AA, Achemlal et al. (in press) Bone mineral density of the spine and femur in healthy Moroccan women. J Clin Densitom 